\title{
Variety theorem for algebras with fuzzy order
}

\author{
Vilem Vychodil* \\ Dept. Computer Science, Palacky University, Olomouc
}

\begin{abstract}
We present generalization of the Bloom variety theorem of ordered algebras in fuzzy setting. We introduce algebras with fuzzy orders which consist of sets of functions which are compatible with particular binary fuzzy relations called fuzzy orders. Fuzzy orders are defined on universe sets of algebras using complete residuated lattices as structures of degrees. In this setting, we show that classes of models of fuzzy sets of inequalities are closed under suitably defined formations of subalgebras, homomorphic images, and direct products. Conversely, we prove that classes having these closure properties are definable by fuzzy sets of inequalities.
\end{abstract}

\section{Introduction}

In this paper, we develop previous results on closure properties of model classes of fuzzy structures [2, 5] which focused on algebras equipped with fuzzy equalities. Recall from [4 that algebras with fuzzy equalities are considered as general algebras, i.e., structures consisting of universe sets equipped with (ordinary) $n$-ary functions, which are in addition compatible with given fuzzy equality relations. Thus, an algebra with fuzzy equality may be viewed as a traditional algebra with an additional relational component. The aim of algebras with fuzzy equalities is to formalize functional systems which preserve similarity in

\footnotetext{
*e-mail: vychodil@binghamton.edu, phone: +420 585634 705, fax: +420 585411643
} 
that the functions, when used with pairwise similar arguments, produce similar results. The existing results on closure properties of algebras with fuzzy equalities include generalization [2] of the Birkhoff variety theorem [8] and results on classes definable by graded implications between identities [5], cf. also [3] for a survey of results.

Our aim in this paper is to study algebras equipped with fuzzy orders which in general are intended to formalize different relationships than similarity fuzzy relations and may be regarded as representations of (graded) preferences. Analogously as in the case of algebras with fuzzy equalities, we assume that functions of algebras are compatible with given fuzzy orders. In this case, the compatibility says that the degree to which the result of a function applied to $b_{1}, \ldots, b_{n}$ is preferred to the result of the function applied to $a_{1}, \ldots, a_{n}$ is at least as high as the degree to which $b_{1}$ is preferred to $a_{1}$ and $\cdots$ and $b_{n}$ is preferred to $a_{n}$ with the logical connective "and" interpreted by a suitable truth function, e.g., a left-continuous triangular norm 23$]$.

One of the important aspects of our approach is that we consider theories as fuzzy sets of atomic formulas (inequalities) prescribing degrees to which the formulas shall be satisfied in models. We thus utilize one of the basic concepts of Pavelka's abstract fuzzy logic [24, 25, 26] in which theories are fuzzy sets of (abstract formulas) and the semantic entailment is defined to degrees, cf. also [18 for a general treatment of related topics. In this setting, we prove that model classes of fuzzy sets of inequalities are closed under formations of homomorphic images, subalgebras, and direct products and, conversely, classes obeying these closure properties are model classes of fuzzy sets of inequalities, establishing an analogy of the Bloom variety theorem [9] for ordered algebras.

This paper is organized as follows. In Section 2 , we present preliminaries. In Section 3 we present the algebras with fuzzy order and their relationship to algebras with fuzzy equalities. Section 4 presents examples of algebras with fuzzy order. Section 5 develops basic algebraic constructions which are further exploited in Section 6 devoted to the relationship of varieties and inequational 
classes of algebras with fuzzy order.

\section{Preliminaries}

A complete (integral commutative) residuated lattice [1, 17] is an algebra $\mathbf{L}=$ $\langle L, \wedge, \vee, \otimes, \rightarrow, 0,1\rangle$ where $\langle L, \wedge, \vee, 0,1\rangle$ is a complete lattice, $\langle L, \otimes, 1\rangle$ is a commutative monoid, and $\otimes$ and $\rightarrow$ satisfy the adjointness property: $a \otimes b \leq c$ iff $a \leq b \rightarrow c(a, b, c \in L)$. One of the important properties implied by the adjointness property is the isotony of $\otimes$. That is, $a \leq b$ and $c \leq d$ yeild $a \otimes c \leq b \otimes d$. Examples of complete residuated lattices include structures on the real unit interval given by left-continuous t-norms [23] as well as finite structures and the structures play important role in fuzzy logics in the narrow sense [15, 20, 21]. A survey of recent results in fuzzy logics can be found in [13, 14].

Given $\mathbf{L}$ and $M \neq \emptyset$, a binary $\mathbf{L}$-relation $R$ on $M$ is a map $R: M \times M \rightarrow L$. For $a, b \in M$, the degree $R(a, b) \in L$ is interpreted as the degree to which $a$ and $b$ are $R$-related. If $\approx, \preccurlyeq, \ldots$ denote binary $\mathbf{L}$-relations, we use the usual infix notation and write $a \approx b$ instead of $\approx(a, b)$ and the like. For binary L-relations $R_{1}$ and $R_{2}$ on $M$, we put $R_{1} \subseteq R_{2}$ whenever $R_{1}(a, b) \leq R_{2}(a, b)$ for all $a, b \in$ $M$ and say that $R_{1}$ is (fully) contained in $R_{2}$. As usual, a binary L-relation $R^{-1}$ satisfying $R^{-1}(a, b)=R(b, a)$ (for all $a, b \in M$ ) is called the inverse of $R$. For convenience, inverses of relations denoted by symbols like $\preccurlyeq, \ldots$ are denoted by $\succcurlyeq, \ldots$ Operations with $\mathbf{L}$-relations can be defined componentwise [1, p. 80] using operations in $\mathbf{L}$. For instance, for an $I$-indexed family $\left\{R_{i} ; i \in I\right\}$ of binary L-relations on $M$, we may consider the intersection $\bigcap_{i \in I} R_{i}$ of all $R_{i}$ $(i \in I)$ which is a binary L-relation with $\left(\bigcap_{i \in I} R_{i}\right)(a, b)=\bigwedge_{i \in I} R_{i}(a, b)$ for all $a, b \in M$.

In the paper, we consider algebras of a given type. A type is given by a set $F$ of function symbols together with their arities. We assume that arity of each $f \in F$ is finite. Recall that an algebra (of type $F$ ) is a structure $\mathbf{M}=\left\langle M, F^{\mathbf{M}}\right\rangle$ where $M$ is a non-empty universe set and $F^{\mathrm{M}}$ is a set of functions interpreting 
the function symbols in F. Namely,

$$
F^{\mathbf{M}}=\left\{f^{\mathbf{M}}: M^{n} \rightarrow M ; f \text { is } n \text {-ary function symbol in } F\right\} .
$$

An algebra with L-equality [4, Definition 3.1] (of type $F$ ) is a structure $\mathbf{M}=$ $\left\langle M, \approx^{\mathbf{M}}, F^{\mathbf{M}}\right\rangle$ such that $\left\langle M, F^{\mathbf{M}}\right\rangle$ is an algebra (of type $F$ ) and $\approx^{\mathbf{M}}$ is a binary L-relation on $M$ satisfying the following conditions:

$$
\begin{gathered}
a \approx^{\mathrm{M}} b=1 \text { iff } a=b, \\
a \approx^{\mathrm{M}} b=b \approx^{\mathrm{M}} a, \\
a \approx^{\mathrm{M}} b \otimes b \approx^{\mathrm{M}} c \leq a \approx^{\mathrm{M}} c, \\
a_{1} \approx^{\mathrm{M}} b_{1} \otimes \cdots \otimes a_{n} \approx^{\mathrm{M}} b_{n} \leq f^{\mathrm{M}}\left(a_{1}, \ldots, a_{n}\right) \approx^{\mathrm{M}} f^{\mathrm{M}}\left(b_{1}, \ldots, b_{n}\right)
\end{gathered}
$$

for all $a, b, c, a_{1}, b_{1}, \ldots, a_{n}, b_{n} \in M$ and any $n$-ary $f \in F$. Recall that condition (1) represents reflexivity and separability, (2) is symmetry, (3) is transitivity with respect to $\otimes$ in $\mathbf{L}$ (so-called $\otimes$-transitivity), and (4) states that $f^{\mathbf{M}}$ is compatible with $\approx^{\mathrm{M}}$ in sense that pairwise similar elements are mapped by $f^{\mathrm{M}}$ to pairwise similar results. Algebraic constructions of algebras with fuzzy equalities are developed in [4, 27, see also [3] for an extensive treatment.

\section{Algebras with Fuzzy Order}

In this section, we introduce algebras with fuzzy order. The motivation is similar as in the case of algebras with fuzzy equalities. The intention is to introduce algebras whose functions are compatible with given fuzzy order. We start by considering algebras with fuzzy equalities as the starting structures and equip them with additional binary L-relations. Later, we observe that the additional L-relations in fact determine the underlying fuzzy equalities.

Definition 1. Let $\mathbf{M}=\left\langle M, \approx^{\mathbf{M}}, F^{\mathbf{M}}\right\rangle$ be an algebra with $\mathbf{L}$-equality. Let $\preccurlyeq^{\mathbf{M}}$ 
be a binary L-relation on $M$ such that

$$
\begin{gathered}
a \preccurlyeq^{\mathrm{M}} a=1, \\
a \preccurlyeq^{\mathrm{M}} b \wedge b \preccurlyeq^{\mathrm{M}} a \leq a \approx^{\mathrm{M}} b, \\
a \preccurlyeq^{\mathrm{M}} b \otimes b \preccurlyeq^{\mathrm{M}} c \leq a \preccurlyeq^{\mathrm{M}} c, \\
a_{1} \approx^{\mathrm{M}} b_{1} \otimes a_{2} \approx^{\mathrm{M}} b_{2} \leq a_{1} \preccurlyeq^{\mathrm{M}} a_{2} \rightarrow b_{1} \preccurlyeq^{\mathrm{M}} b_{2}, \\
a_{1} \preccurlyeq^{\mathrm{M}} b_{1} \otimes \cdots \otimes a_{n} \preccurlyeq^{\mathrm{M}} b_{n} \leq f^{\mathrm{M}}\left(a_{1}, \ldots, a_{n}\right) \preccurlyeq^{\mathrm{M}} f^{\mathrm{M}}\left(b_{1}, \ldots, b_{n}\right),
\end{gathered}
$$

for all $a, b, c, a_{1}, b_{1}, \ldots, a_{n}, b_{n} \in M$ and any $n$-ary $f \in F$. The structure $\left\langle M, \approx^{\mathbf{M}}, \preccurlyeq^{\mathbf{M}}, F^{\mathbf{M}}\right\rangle$ is called an algebra with $\mathbf{L}$-equality and $\mathbf{L}$-order.

As in the case of algebras with fuzzy equalities, (5) states that $\preccurlyeq^{\mathrm{M}}$ is reflexive, (6) represents antisymetry of $\preccurlyeq^{\mathrm{M}}$ with respect to $\approx^{\mathrm{M}}$, (7) is $\otimes$-transitivity, and (9) is compatibility of $f^{\mathrm{M}}$ with $\preccurlyeq^{\mathrm{M}}$. Put in words, (9) states that the degree to which $f^{\mathrm{M}}\left(b_{1}, \ldots, b_{n}\right)$ is greater than (or equal to) $f^{\mathrm{M}}\left(a_{1}, \ldots, a_{n}\right)$ is at least the degree to which $b_{1}$ is greater (or equal to) than $a_{1}$ and $\cdots$ and $b_{n}$ is greater (or equal to) than $a_{n}$ with "and" interpreted by $\otimes$. Using the adjointness, 8) can be equivalently restated as

$$
a_{1} \approx^{\mathrm{M}} b_{1} \otimes a_{2} \approx^{\mathrm{M}} b_{2} \otimes a_{1} \preccurlyeq^{\mathrm{M}} a_{2} \leq b_{1} \preccurlyeq^{\mathrm{M}} b_{2}
$$

and the condition expresses that $\preccurlyeq^{\mathbf{M}}$ is compatible with $\approx^{\mathbf{M}}$. It can be shown that the conditions (6) and (8) we have used together represent a constraint on $\approx^{\mathbf{M}}$ with respect to $\preccurlyeq^{\mathbf{M}}$. Namely, $\approx^{\mathbf{M}}$ in $\mathbf{M}$ is uniquely given by $\preccurlyeq^{\mathbf{M}}$ as it is shown by the following assertion.

Theorem 2. Let $\mathbf{M}=\left\langle M, \approx^{\mathbf{M}}, F^{\mathbf{M}}\right\rangle$ be an algebra with $\mathbf{L}$-equality and let $\preccurlyeq^{\mathbf{M}}$ be a binary $\mathbf{L}$-relation on $M$ satisfying (5), (6), (7), and (9). Then the following conditions are equivalent:

(i) $\left\langle M, \approx^{\mathbf{M}}, \preccurlyeq^{\mathbf{M}}, F^{\mathbf{M}}\right\rangle$ is an algebra with $\mathbf{L}$-equality and $\mathbf{L}$-order;

$($ ii $) \approx^{\mathrm{M}}$ and $\preccurlyeq^{\mathrm{M}}$ satisfy (8);

(iii) $\approx^{\mathrm{M}} \subseteq \preccurlyeq^{\mathrm{M}} ;$ 
$(i v) \approx^{\mathrm{M}}=\preccurlyeq^{\mathrm{M}} \cap \succcurlyeq^{\mathrm{M}} ;$

$(v) \approx^{\mathbf{M}}$ is the symmetric interior of $\preccurlyeq^{\mathbf{M}}$.

Proof. " $(i) \Rightarrow($ ii $) "$ : Trivial.

"(ii) $\Rightarrow($ iii $) ":$ As a particular case of (8) for $a_{1}=a_{2}=b_{1}$, we have

$$
b_{1} \approx^{\mathrm{M}} b_{1} \otimes b_{1} \approx^{\mathrm{M}} b_{2} \otimes b_{1} \preccurlyeq^{\mathrm{M}} b_{1} \leq b_{1} \preccurlyeq^{\mathrm{M}} b_{2} .
$$

Now, using (1) and (5), the previous inequality yields

$$
b_{1} \approx^{\mathbf{M}} b_{2}=1 \otimes b_{1} \approx^{\mathbf{M}} b_{2} \otimes 1 \leq b_{1} \preccurlyeq^{\mathbf{M}} b_{2},
$$

proving ( $i i i)$.

"(iii) $\Rightarrow($ iv $) ":$ Take any $a, b \in M$. Since $\approx^{\mathrm{M}}$ satisfies (2), using the assumption $\approx^{\mathbf{M}} \subseteq \preccurlyeq^{\mathbf{M}}$, we get

$$
a \approx^{\mathrm{M}} b=b \approx^{\mathrm{M}} a \leq b \preccurlyeq^{\mathrm{M}} a=a \succcurlyeq^{\mathrm{M}} b
$$

which yields $\approx^{\mathrm{M}} \subseteq \succcurlyeq^{\mathrm{M}}$. Hence, $\approx^{\mathrm{M}} \subseteq \preccurlyeq^{\mathrm{M}} \cap \succcurlyeq^{\mathrm{M}}$. The converse inclusion follows directly by (6).

"(iv) $\Rightarrow(v) "$ : Follows by properties of interior systems and interior operators. In a more detail, $\preccurlyeq^{\mathrm{M}} \cap \succcurlyeq^{\mathrm{M}}$ is obviously symmetric and it is a subset of $\preccurlyeq^{\mathrm{M}}$. If $R$ is symmetric and $R \subseteq \preccurlyeq^{\mathbf{M}}$, then the symmetry of $R$ gives $R \subseteq \succcurlyeq^{\mathrm{M}}$ and thus $R \subseteq \preccurlyeq^{\mathrm{M}} \cap \succcurlyeq^{\mathrm{M}}$, proving that $\preccurlyeq^{\mathrm{M}} \cap \succcurlyeq^{\mathrm{M}}$ is the symmetric interior of $\preccurlyeq^{\mathrm{M}}$.

" $(v) \Rightarrow(i) ":$ It suffices to check that $\approx^{\mathbf{M}}$ and $\preccurlyeq^{\mathbf{M}}$ satisfy (8). The fact that $\approx^{\mathrm{M}}$ is the symmetric interior of $\preccurlyeq^{\mathrm{M}}$ yields $\approx^{\mathrm{M}} \subseteq \preccurlyeq^{\mathrm{M}}$ and $\approx^{\mathrm{M}} \subseteq \succcurlyeq^{\mathrm{M}}$. Therefore, using the assumption and (7) twice we get

$$
\begin{aligned}
a_{1} \approx^{\mathrm{M}} b_{1} \otimes a_{2} \approx^{\mathrm{M}} b_{2} \otimes a_{1} \preccurlyeq^{\mathrm{M}} a_{2} & \leq b_{1} \preccurlyeq^{\mathrm{M}} a_{1} \otimes a_{2} \preccurlyeq^{\mathrm{M}} b_{2} \otimes a_{1} \preccurlyeq^{\mathrm{M}} a_{2} \\
& \leq b_{1} \preccurlyeq^{\mathrm{M}} b_{2}
\end{aligned}
$$

which proves (8).

Remark 1. Note that [1, Definition 4.42] introduces the notion of an $\mathbf{L}$-ordered set as a set $M$ equipped with $\approx^{\mathrm{M}}$ and $\preccurlyeq^{\mathrm{M}}$ satisfying (5), (6), (7), and (10). 
Hence, algebras with fuzzy equalities and fuzzy orders introduced by Definition 1 are $\mathbf{L}$-ordered sets in sense of [1] with an additional compatible functional component. Note also that [1, Lemma 4.45] shows that L-ordered sets satisfy the condition (iv) in Theorem 2, The notion of an $\mathbf{L}$-order we utilize in this paper is not the only one generalization of a partial order in graded setting. Graded order relations or fuzzy orders are often defined so that the antisymmetry condition (6) is formulated using $\otimes$ in place of $\wedge$, see [10].

In our approach to algebras with fuzzy equalities and fuzzy orders, we have started with algebras with fuzzy equalities and expanded the structures by an additional binary fuzzy relation. The following theorem shows that we may as well start with $\preccurlyeq^{\mathrm{M}}$ satisfying certain conditions and consider $\approx^{\mathrm{M}}$ a derived fuzzy relation.

Theorem 3. Let $\mathbf{M}=\left\langle M, F^{\mathbf{M}}\right\rangle$ be an algebra and $\preccurlyeq^{\mathbf{M}}$ be a binary $\mathbf{L}$-relation on $M$ satisfying (5), (7), (9). If for any distinct elements $a, b \in M$ we have $a \preccurlyeq^{\mathbf{M}} b \neq 1$ or $b \preccurlyeq^{\mathbf{M}} a \neq 1$, then $\left\langle M, \approx^{\mathbf{M}}, \preccurlyeq^{\mathbf{M}}, F^{\mathbf{M}}\right\rangle$ with $\approx^{\mathbf{M}}=\preccurlyeq^{\mathbf{M}} \cap \succcurlyeq^{\mathbf{M}}$ is an algebra with $\mathbf{L}$-equality and $\mathbf{L}$-order.

Proof. First, we show that $\left\langle M, \approx^{\mathbf{M}}, F^{\mathbf{M}}\right\rangle$ is an algebra with L-equality. Since $\approx^{\mathrm{M}}=\preccurlyeq^{\mathrm{M}} \cap \succcurlyeq^{\mathrm{M}}$, we get that $\approx^{\mathrm{M}}$ satisfies (2). If $a=b$ then (5) yields $a \approx^{\mathbf{M}} b=1$. Conversely, if $a \neq b$, then $a \preccurlyeq^{\mathbf{M}} b \neq 1$ or $b \preccurlyeq^{\mathbf{M}} a \neq 1$, i.e., $a \approx^{\mathrm{M}} b \neq 1$. Altogether, $\approx^{\mathrm{M}}$ satisfies (1). Moreover, $\approx^{\mathrm{M}}$ is $\otimes$-transitive. Indeed, for $a, b, c \in M$, using (7), we have

$$
a \approx^{\mathrm{M}} b \otimes b \approx^{\mathrm{M}} c \leq a \preccurlyeq^{\mathrm{M}} b \otimes b \preccurlyeq^{\mathrm{M}} c \leq a \preccurlyeq^{\mathrm{M}} c
$$

and

$$
a \approx^{\mathrm{M}} b \otimes b \approx^{\mathrm{M}} c \leq b \preccurlyeq^{\mathrm{M}} a \otimes c \preccurlyeq^{\mathrm{M}} b \leq c \preccurlyeq^{\mathrm{M}} a
$$

and thus

$$
a \approx^{\mathrm{M}} b \otimes b \approx^{\mathrm{M}} c \leq a \preccurlyeq^{\mathrm{M}} c \wedge c \preccurlyeq^{\mathrm{M}} a=a \approx^{\mathrm{M}} c
$$


which proves (3). In a similar way, we prove that (4) is satisfied. Using (9),

$$
\begin{aligned}
a_{1} \approx^{\mathrm{M}} b_{1} \otimes \cdots \otimes a_{n} \approx^{\mathrm{M}} b_{n} & \leq a_{1} \preccurlyeq^{\mathrm{M}} b_{1} \otimes \cdots \otimes a_{n} \preccurlyeq^{\mathrm{M}} b_{n} \\
& \leq f^{\mathrm{M}}\left(a_{1}, \ldots, a_{n}\right) \preccurlyeq^{\mathrm{M}} f^{\mathrm{M}}\left(b_{1}, \ldots, b_{n}\right) .
\end{aligned}
$$

And analogously for $\preccurlyeq$ replaced by $\succcurlyeq$ which together yield (4). As a consequence, $\left\langle M, \approx^{\mathbf{M}}, F^{\mathbf{M}}\right\rangle$ is an algebra with $\mathbf{L}$-equality. Now, in order to see that $\left\langle M, \approx^{\mathbf{M}}, \preccurlyeq^{\mathbf{M}}, F^{\mathbf{M}}\right\rangle$ is an algebra with $\mathbf{L}$-equality and $\mathbf{L}$-order, observe that (6) follows directly by $\approx^{\mathrm{M}}=\preccurlyeq^{\mathrm{M}} \cap \succcurlyeq^{\mathrm{M}}$ and apply Theorem 2 .

By virtue of Theorem 3, we may call an algebra $\mathbf{M}=\left\langle M, \approx^{\mathbf{M}}, \preccurlyeq^{\mathbf{M}}, F^{\mathbf{M}}\right\rangle$ with $\mathbf{L}$-equality and $\mathbf{L}$-order simply an algebra with $\mathbf{L}$-order and write

$$
\mathbf{M}=\left\langle M, \preccurlyeq^{\mathbf{M}}, F^{\mathbf{M}}\right\rangle .
$$

Whenever we do so, we consider $\approx^{\mathrm{M}}$ to be the symmetric interior of $\preccurlyeq^{\mathrm{M}}$.

\section{Examples}

In this section, we show examples of algebras with fuzzy order. We focus on examples of structures that are used further in the paper as well as strucures that naturally appear in fuzzy relational systems.

Example 1. Recall that in the classic case, each algebra can be expanded to an ordered algebra by considering identity as the order. Such ordered algebras are usually called discrete. In our case, for each algebra $\left\langle M, \approx^{\mathbf{M}}, F^{\mathbf{M}}\right\rangle$ with L-equality $\approx^{\mathbf{M}}$ we may consider $\mathbf{M}=\left\langle M, \approx^{\mathbf{M}}, \preccurlyeq^{\mathbf{M}}, F^{\mathbf{M}}\right\rangle$ where $\preccurlyeq^{\mathbf{M}}=\approx^{\mathbf{M}}$. Observe that $\mathbf{M}$ is indeed an algebra with $\mathbf{L}$-order since (5) follows from (1), (6) is satisfied trivially, (7) becomes (3), (9) becomes (4), and then (8) follows by Theorem 2 As an important consequence, each algebra with L-equality can be expanded into an algebra with $\mathbf{L}$-order.

Example 2. There are two important special cases of algebras with $\mathbf{L}$-orders which result as in Example 1. First, $\mathbf{M}=\left\langle M, \approx^{\mathbf{M}}, \preccurlyeq^{\mathbf{M}}, F^{\mathbf{M}}\right\rangle$ is called trivial 
if $M=\{\emptyset\}$, and both $\approx^{\mathrm{M}}$ and $\preccurlyeq^{\mathrm{M}}$ are identities. That is, the universe $M$ of $\mathbf{M}$ consists of a single element (the empty set $\emptyset$ ) and $\emptyset \approx^{\mathbf{M}} \emptyset=\emptyset \preccurlyeq^{\mathbf{M}} \emptyset=1$. Second, let $X$ be a set of variables and let $T(X)$ denote the set of all terms in variables $X$ (using function symbols in $F$ ). If $T(X) \neq \emptyset$ (e.g., if $F$ contains a nullary function symbol or $X$ is non-empty), we denote by $\mathbf{T}(X)$ the algebra $\left\langle T(X), \approx^{\mathbf{T}(X)}, \preccurlyeq^{\mathbf{T}(X)}, F^{\mathbf{T}(X)}\right\rangle$ with $\mathbf{L}$-equality and $\mathbf{L}$-order where both $\approx^{\mathbf{M}}$ and $\preccurlyeq^{\mathrm{M}}$ are identities, i.e.,

$$
t \preccurlyeq^{\mathbf{T}(X)} t^{\prime}= \begin{cases}1, & \text { if } t=t^{\prime}, \\ 0, & \text { otherwise, }\end{cases}
$$

and analogously for $\approx^{\mathbf{T}(X)}$. Thus, $\mathbf{T}(X)$ results from an ordinary term algebra by adding $\approx^{\mathbf{T}(X)}$ and $\preccurlyeq^{\mathbf{T}(X)}$, both being identity L-relations on $\mathbf{T}(X)$. We therefore call $\mathbf{T}(X)$ the (absolutely free) term algebra with $\mathbf{L}$-order over variables in $X$. We shall see later in the paper that the trivial and term algebras are two borderline cases among algebras with $\mathbf{L}$-order (of the same type) in terms of the satisfaction of inequlities and play analogous roles as their classic counterparts.

In the next example, we intend to demonstrate that algebras with $\mathbf{L}$-orders may naturally appear as structures describing entailment degrees of graded ifthen formulas. For the purpose of illustration, we briefly recall fuzzy attribute implications (FAIs) and their semantic entailment [7. Consider a non-empty set $Y$ the elements of which are called attributes. Each map $A: Y \rightarrow L$ (an $\mathbf{L}$-set in $Y$ in the usual terminology of fuzzy relational systems [1, 19]) prescribes a degree $A(y) \in L$ to each attribute $y \in Y$. The degree $A(y)$ is interpreted as "a degree to which an object has attribute $y$ ", i.e., $A$ may be interpreted as a collection of graded attributes of an object. The set of all maps of the form $A: Y \rightarrow L$ is denoted by $L^{Y}$.

For $A, B \in L^{Y}$, we may introduce a degree to which $A$ is included in $B$ by

$$
S(A, B)=\bigwedge_{y \in Y}(A(y) \rightarrow B(y)),
$$

where $\rightarrow$ is the residuum in $\mathbf{L}$. A fuzzy (or graded) attribute implication (shortly, a FAI) is any expression of the from $A \Rightarrow B$, where $A, B \in L^{Y}$. The degree to 
which $A \Rightarrow B$ is true in $M \in L^{Y}$, written $\|A \Rightarrow B\|_{M}$ is defined using (12) as follows:

$$
\|A \Rightarrow B\|_{M}=S(A, M) \rightarrow S(B, M)
$$

Assuming that $M$ is a collection of graded attributes of an object $x$, we may interpret $\|A \Rightarrow B\|_{M}$ as the degree to which it is true that "if $x$ has all the (graded) attributes from $A$, then $x$ has all the (graded) attributes from $B$ ". Therefore, graded attribute implications describe if-then dependencies between graded attributes of objects. Let us note that the approach in [7] is more general in that the definition $(13)$ is parameterized by a linguistic hedge [6, 16, 28, but we omit this technical issue for the sake of simplicity.

One of the basic problems related to FAIs is semantic entailment of FAIs from (graded) collections of other FAIs, its characterization, axiomatization, and other related problems, cf. [7]. Denote the set of all FAIs (in $Y$ ) by $F m l$ and let $T$ be a map $T: F m l \rightarrow L$. Each $T(A \Rightarrow B)$ may be seen as a degree to which $A \Rightarrow B$ is prescribed by $T$. We put

$$
\operatorname{Mod}(T)=\left\{M \in L^{Y} ; T(A \Rightarrow B) \leq\|A \Rightarrow B\|_{T} \text { for all } A, B \in L^{Y}\right\}
$$

and call $\operatorname{Mod}(T)$ the set of all models of $T$. Moreover, we put

$$
\|A \Rightarrow B\|_{T}=\bigwedge_{M \in \operatorname{Mod}(T)}\|A \Rightarrow B\|_{M}
$$

and call $\|A \Rightarrow B\|_{T}$ the degree to which $A \Rightarrow B$ is semantically entailed by $T$. Note that the way we have defined (15) conforms to the graded semantics of formulas as it was proposed by Pavelka [24, 25, 26, cf. also [18] and [21, Section 9.2]. The next example shows that the entailment degrees may be represented by an algebra with $\mathbf{L}$-order which is related to 15 .

Example 3. Assuming that $T$ is a set of FAIs in $Y$, we may introduce an algebra of classes of semantically equivalent elements in $L^{Y}$ with the $\mathbf{L}$-order corresponding to 15 . First, we put

- $L^{Y} / T=\left\{[A]_{T} ; A \in L^{Y}\right\}$, where 
- $[A]_{T}=\left\{B \in L^{Y} ;\|A \Rightarrow B\|_{T}=\|B \Rightarrow A\|_{T}=1\right\}$.

Obviously, $L^{Y} / T$ is a partition of $L^{Y}$ and each $[A]_{T} \in L^{Y} / T$ contains all $B \in L^{Y}$ which are equivalent to $A$ under $T$. Now, consider

$$
\mathbf{M}_{T}=\left\langle L^{Y} / T, \preccurlyeq \mathbf{M}_{T}, \cup^{\mathbf{M}_{T}}, 0^{\mathbf{M}_{T}}, 1^{\mathbf{M}_{T}}\right\rangle,
$$

where $0^{\mathbf{M}_{T}}=\left[0_{Y}\right]_{T}\left(\right.$ with $0_{Y}(y)=0$ for all $\left.y \in Y\right), 1^{\mathbf{M}_{T}}=\left[1_{Y}\right]_{T}$ (with $1_{Y}(y)=1$ for all $\left.y \in Y\right),[A]_{T} \cup \mathbf{M}_{T}[B]_{T}=[A \cup B]_{T}$, and

$$
[A]_{T} \preccurlyeq^{\mathbf{M}_{T}}[B]_{T}=\|A \Rightarrow B\|_{T} .
$$

We claim that $\mathbf{M}_{T}$ is an algebra with $\mathbf{L}$-order. In order to see that, we have to check that $\cup^{\mathbf{M}_{T}}$ and $\preccurlyeq \mathbf{M}_{T}$ are well defined and that all conditions required by Theorem 3 hold. The fact that $\cup \mathbf{M}$ is well defined follows directly by the facts that $S(A, M) \wedge S(B, M)=S(A \cup B, M)$ is true for all $A, B, M \in L^{Y}$ and that $\|A \Rightarrow B\|_{T}=1$ means $S(A, M) \leq S(B, M)$ for each $M \in \operatorname{Mod}(T)$. In case of $\preccurlyeq \mathbf{M}_{T}$, we show the argument in a more detail: For $A^{\prime} \in[A]_{T}$ and $B^{\prime} \in[B]_{T}$, we have $\left\|A^{\prime} \Rightarrow A\right\|_{T}=1$ and $\left\|B \Rightarrow B^{\prime}\right\|_{T}=1$ from which it follows that

$$
\begin{aligned}
1 \otimes\|A \Rightarrow B\|_{T} \otimes 1 & =\left\|A^{\prime} \Rightarrow A\right\|_{T} \otimes\|A \Rightarrow B\|_{T} \otimes\left\|B \Rightarrow B^{\prime}\right\|_{T} \\
& \leq\left\|A^{\prime} \Rightarrow B^{\prime}\right\|_{T},
\end{aligned}
$$

because

$$
\begin{aligned}
\|A \Rightarrow B\|_{T} \otimes\|B \Rightarrow C\|_{T} & \leq \bigwedge_{M \in \operatorname{Mod}(T)}\left(\|A \Rightarrow B\|_{M} \otimes\|B \Rightarrow C\|_{M}\right) \\
& \leq \bigwedge_{M \in \operatorname{Mod}(T)}(S(A, M) \rightarrow S(C, M)) \\
& =\|A \Rightarrow C\|_{T}
\end{aligned}
$$

is true for all $A, B, C \in L^{Y}$. Analogously, we prove $\left\|A^{\prime} \Rightarrow B^{\prime}\right\|_{T} \leq\|A \Rightarrow B\|_{T}$. As a consequence, $\preccurlyeq \mathbf{M}_{T}$ is well defined. Furthermore, (5) holds because $\| A \Rightarrow$ $A \|_{T}=1$ is true and (7) is a consequence of (16). In order to check (9), it suffices to show that

$$
\|A \Rightarrow B\|_{T} \otimes\|C \Rightarrow D\|_{T} \leq\|A \cup C \Rightarrow B \cup D\|_{T}
$$


which is indeed the case: This follows by similar arguments as before, utilizing the fact that $(a \rightarrow b) \otimes(c \rightarrow d) \leq(a \wedge c) \rightarrow(b \wedge d)$ holds for all $a, b, c, d \in L$ in any residuated lattice [17. Altogether, Theorem 3 yields that $\mathbf{M}_{T}$ is an algebra with $\mathbf{L}$-order and the induced $\mathbf{L}$-equality is

$$
[A]_{T} \approx^{\mathbf{M}_{T}}[B]_{T}=\|A \Rightarrow B\|_{T} \wedge\|B \Rightarrow A\|_{T}
$$

Therefore, $\mathbf{M}_{T}$ may be understood as an algebraic representation of the semantic closure of $T$ with $[A]_{T} \approx \mathbf{M}_{T}[B]_{T}$ being the degree to which $A$ and $B$ are equivalent under $T$ and $[A]_{T} \preccurlyeq \mathbf{M}_{T}[B]_{T}$ being the degree to which $B$ follows from $A$ under $T$.

Note that $\mathbf{M}_{T}$ can be further extended, e.g., by considering unary operations $\otimes^{a}(a \in L)$ defined by $\otimes^{a}\left([A]_{T}\right)=[a \otimes A]_{T}$ where $a \otimes A \in L^{Y}$ is defined by $(a \otimes A)(y)=a \otimes A(y)$ for all $y \in Y$. Interestingly, structures like $\mathbf{M}_{T}$ can also be defined based on syntactic entailment of FAIs and can be regarded as particular Lindenbaum algebras with fuzzy order. We refrain from further details because we do not want to digress too much from the main subject of this paper which are properties of classes of general structures.

\section{$5 \quad$ Algebraic Constructions}

In this section, we summarize the basic algebraic constructions essential for establishing an analogy of the variety theorem in Section 6. The constructions generalize the algebraic constructions presented in 4 4 in a way that by omitting the fuzzy order, we get exactly the algebraic constructions with algebras with fuzzy equalities as they have been proposed and described in [4]. Detailed description of operations for ordinary algebras and algebras with L-equalities and can be found in [3, 11].

\section{Subalgebras}

Let $\mathbf{M}=\left\langle M, \approx^{\mathbf{M}}, \preccurlyeq^{\mathbf{M}}, F^{\mathbf{M}}\right\rangle$ be an algebra with $\mathbf{L}$-order. A subuniverse $N$ of $\mathbf{M}$ is a subset of $M$ which is closed under all functions from $F^{\mathbf{M}}$. If $N \neq \emptyset$ is a 
subuniverse of $\mathbf{M}$, then $\mathbf{N}=\left\langle N, \approx^{\mathbf{N}}, \preccurlyeq^{\mathbf{N}}, F^{\mathbf{N}}\right\rangle$ where

- $\approx^{\mathbf{N}}$ is restriction of $\approx^{\mathrm{M}}$ to $N$ (i.e., $a \approx^{\mathbf{N}} b=a \approx^{\mathrm{M}} b$ for all $a, b \in N$ );

- $\preccurlyeq^{\mathbf{N}}$ is restriction of $\preccurlyeq^{\mathbf{M}}$ to $N$ (i.e., $a \preccurlyeq^{\mathbf{N}} b=a \preccurlyeq^{\mathbf{M}} b$ for all $a, b \in N$ );

- each $f^{\mathbf{N}}$ is a restriction of $f^{\mathbf{M}}$ to $N$ (i.e., $f^{\mathbf{N}}\left(a_{1}, \ldots, a_{n}\right)=f^{\mathbf{M}}\left(a_{1}, \ldots, a_{n}\right)$ for all $\left.a_{1}, \ldots, a_{n} \in N\right)$

is called a subalgebra of $\mathbf{M}$.

\section{Direct Products}

Let $\mathbf{M}_{i}(i \in I)$ be an $I$-indexed family of algebras with $\mathbf{L}$-order (of the same type $F)$. A direct product $\prod_{i \in I} \mathbf{M}_{i}$ of $\mathbf{M}_{i}(i \in I)$ is an algebra with $\mathbf{L}$ order $\prod_{i \in I} \mathbf{M}_{i}=\left\langle\prod_{i \in I} M_{i}, \preccurlyeq \prod_{i \in I} \mathbf{M}_{i}, F \prod_{i \in I} \mathbf{M}_{i}\right\rangle$ defined on the direct product $\prod_{i \in I} M_{i}$ of the universe sets of all $\mathbf{M}_{i}$, where the operations are defined componentwise by

$$
f^{\prod_{i \in I} \mathbf{M}_{i}}\left(a_{1}, \ldots, a_{n}\right)(i)=f^{\mathbf{M}_{i}}\left(a_{1}(i), \ldots, a_{n}(i)\right)
$$

for any $n$-ary $f \in F, a_{1}, \ldots, a_{n} \in \prod_{i \in I} M_{i}$, and $i \in I$; and $\preccurlyeq \prod_{i \in I} \mathbf{M}_{i}$ is introduced as follows:

$$
a \preccurlyeq \prod_{i \in I} \mathbf{M}_{i} b=\bigwedge_{i \in I} a(i) \preccurlyeq \mathbf{M} b(i),
$$

for all $a, b \in \prod_{i \in I} M_{i}$. It it easily seen that $\prod_{i \in I} \mathbf{M}_{i}$ is indeed an algebra with L-order since it satisfies the conditions of Theorem 3 . As a consequence, for the corresponding $\mathbf{L}$-equality $\approx \prod_{i \in I} \mathbf{M}_{i}$, we get

$$
a \approx \prod_{i \in I} \mathbf{M}_{i} b=\bigwedge_{i \in I} a(i) \approx^{\mathbf{M}_{i}} b(i)
$$

Analogously as in the ordinary setting, the direct product of an empty family of algebras with $\mathbf{L}$-order is the trivial algebra with $\mathbf{L}$-order, see Example 2. 


\section{Homomorphisms and Factor Algebras}

Let $\mathbf{M}$ and $\mathbf{N}$ be algebras with $\mathbf{L}$-orders (of the same type $F$ ). A map $h: M \rightarrow N$ which satisfies equality

$$
h\left(f^{\mathrm{M}}\left(a_{1}, \ldots, a_{n}\right)\right)=f^{\mathbf{N}}\left(h\left(a_{1}\right), \ldots, h\left(a_{n}\right)\right)
$$

for any $n$-ary $f \in F$ and all $a_{1}, \ldots, a_{n} \in M$; and

$$
a \preccurlyeq^{\mathrm{M}} b \leq h(a) \preccurlyeq^{\mathbf{N}} h(b)
$$

for all $a, b \in M$ is called a homomorphism and is denoted by $h: \mathbf{M} \rightarrow \mathbf{N}$. Therefore, homomorphisms are maps which are compatible with the functional parts of $\mathbf{M}$ and $\mathbf{N}$ and the $\mathbf{L}$-orders of $\mathbf{M}$ and $\mathbf{N}$. As a consequence, we get that homomorphisms are also compatible with $\mathbf{L}$-equalities of $\mathbf{M}$ and $\mathbf{N}$ since for any $a, b \in M$, it follows that

$$
a \approx^{\mathrm{M}} b \leq h(a) \approx^{\mathrm{N}} h(b) .
$$

A homomorphism $h: \mathbf{M} \rightarrow \mathbf{N}$ is called an embedding whenever

$$
a \preccurlyeq^{\mathrm{M}} b=h(a) \preccurlyeq^{\mathrm{N}} h(b)
$$

holds for all $a, b \in M$. If $h: \mathbf{M} \rightarrow \mathbf{N}$ is an embedding then

$$
a \approx^{\mathbf{M}} b=h(a) \approx^{\mathbf{N}} h(b)
$$

for any $a, b \in M$, which is easy to see, cf. [1, Lemma 4.46]. If $h: \mathbf{M} \rightarrow \mathbf{N}$ is surjective, then $\mathbf{N}$ is called a (homomorphic) image of $\mathbf{M}$. As usual, surjective embeddings may be called isomorphisms.

Remark 2. Note that we may consider a category consisting of algebras with $\mathbf{L}$ orders as objects, homomorphisms of algebras with $\mathbf{L}$-orders as arrows, with the composition $\circ$ being the usual composition of maps between sets, and identity arrows being the usual identity maps. That is, for $f: \mathbf{M} \rightarrow \mathbf{N}$ and $g: \mathbf{N} \rightarrow \mathbf{P}$, we put $(f \circ g)(a)=g(f(a))$ (written in the diagrammatic notation) for all $a \in M$. Clearly, $f \circ g: \mathbf{M} \rightarrow \mathbf{P}$ is a homomorphism of algebras with $\mathbf{L}$-orders. 
Let us note that surjective homomorphisms are exactly the epic arrows and embeddings are monic but there are monic arrows which are not embeddings.

In the paper, we further utilize the fact that homomorphic images may be represented by factor algebras with $\mathbf{L}$-orders. Consider an algebra $\mathbf{M}$ with $\mathbf{L}$ order. A binary $\mathbf{L}$-relation $\xi$ on $M$ is called an $\mathbf{L}$-preorder compatible with $\mathbf{M}$ whenever it satisfies

$$
\begin{aligned}
\preccurlyeq^{\mathrm{M}} & \subseteq \xi, \\
\xi(a, b) \otimes \xi(b, c) & \leq \xi(a, c), \\
\xi\left(a_{1}, b_{1}\right) \otimes \cdots \otimes \xi\left(a_{n}, b_{n}\right) & \leq \xi\left(f^{\mathrm{M}}\left(a_{1}, \ldots, a_{n}\right), f^{\mathrm{M}}\left(b_{1}, \ldots, b_{n}\right)\right),
\end{aligned}
$$

for all $a, b, c, a_{1}, b_{1}, \ldots, a_{n}, b_{n} \in M$ and any $n$-ary $f \in F$. It can be shown that compatible $\mathbf{L}$-preorders on $\mathbf{M}=\left\langle M, \approx^{\mathbf{M}}, \preccurlyeq^{\mathbf{M}}, F^{\mathbf{M}}\right\rangle$ induce congruences [4, Definition 3.3] on $\left\langle M, \approx^{\mathbf{M}}, F^{\mathbf{M}}\right\rangle$. Indeed, for $\theta$ being the symmetric interior of $\xi$, i.e., for $\theta=\xi \cap \xi^{-1}$, we have

$$
\begin{aligned}
\approx^{\mathrm{M}} & \subseteq \theta, \\
\theta(a, b) & =\theta(b, a), \\
\theta(a, b) \otimes \theta(b, c) & \leq \theta(a, c), \\
\theta\left(a_{1}, b_{1}\right) \otimes \cdots \otimes \theta\left(a_{n}, b_{n}\right) & \leq \theta\left(f^{\mathrm{M}}\left(a_{1}, \ldots, a_{n}\right), f^{\mathrm{M}}\left(b_{1}, \ldots, b_{n}\right)\right) .
\end{aligned}
$$

Hence, according to [4, we may consider a factor algebra with $\mathbf{L}$-equality $\mathbf{M} / \theta=$ $\left\langle M / \theta, \approx^{\mathrm{M} / \theta}, F^{\mathrm{M} / \theta}\right\rangle$ as follows:

- $M / \theta=\left\{[a]_{\theta} ; a \in M\right\}$ where $[a]_{\theta}=\{b \in M ; \theta(a, b)=1\}$;

- $f^{\mathrm{M} / \theta}\left(\left[a_{1}\right]_{\theta}, \ldots,\left[a_{n}\right]_{\theta}\right)=\left[f^{\mathrm{M}}\left(a_{1}, \ldots, a_{n}\right)\right]_{\theta}$;

- $[a]_{\theta} \approx^{\mathrm{M} / \theta}[b]_{\theta}=\theta(a, b)$.

That is, $\mathbf{M} / \theta$ can be seen as an ordinary factor algebra $\left\langle M, F^{\mathbf{M}}\right\rangle$ modulo $\{\langle a, b\rangle ; \theta(a, b)=1\}$ which is in addition equipped with $\approx^{\mathrm{M} / \theta}$ defined as above. We put $M / \xi=M / \theta,[a]_{\xi}=[a]_{\theta}, f^{\mathrm{M} / \xi}=f^{\mathrm{M} / \theta}, \approx^{\mathrm{M} / \xi}=\approx^{\mathrm{M} / \theta}$ and define 
$\preccurlyeq^{\mathrm{M} / \xi}$ by

$$
[a]_{\xi} \preccurlyeq^{\mathrm{M} / \xi}[b]_{\xi}=\xi(a, b) .
$$

The resulting structure $\mathbf{M} / \xi=\left\langle M / \xi, \approx^{\mathbf{M} / \xi}, \preccurlyeq^{\mathbf{M} / \xi}, F^{\mathbf{M} / \xi}\right\rangle$ is called a factor algebra with $\mathbf{L}$-order of $\mathbf{M}$ modulo $\xi$. The following lemma shows that $\mathbf{M} / \xi$ is indeed an algebra with L-order:

Lemma 4. $\preccurlyeq^{\mathrm{M} / \xi}$ defined by (32) is well defined and satisfies (5)-(9) with respect to $\approx^{\mathbf{M} / \xi}$. As a consequence, $\mathbf{M} / \xi$ is a well-defined algebra with $\mathbf{L}$-order.

Proof. We first show that the value of $[a]_{\xi} \preccurlyeq^{\mathbf{M} / \xi}[b]_{\xi}$ does not depend on the choice of elements from either class. Thus, take $a^{\prime} \in[a]_{\xi}$ and $b^{\prime} \in[b]_{\xi}$. By definition, we get $\theta\left(a, a^{\prime}\right)=1$ for $\theta=\xi \cap \xi^{-1}$ and thus $\xi\left(a, a^{\prime}\right)=1$ and $\xi\left(a^{\prime}, a\right)=1$. Analogously, $\xi\left(b, b^{\prime}\right)=1$ and $\xi\left(b^{\prime}, b\right)=1$. Applying 26 twice, we get

$$
1 \otimes 1 \otimes \xi(a, b)=\xi\left(a^{\prime}, a\right) \otimes \xi\left(b, b^{\prime}\right) \otimes \xi(a, b) \leq \xi\left(a^{\prime}, b^{\prime}\right)
$$

and analogously $\xi\left(a^{\prime}, b^{\prime}\right) \leq \xi(a, b)$, meaning $\xi(a, b)=\xi\left(a^{\prime}, b^{\prime}\right)$, i.e., (32) is well defined. Now, observe that (5) follows by (25). Moreover, (6) follows by the fact that

$$
\begin{aligned}
{[a]_{\xi} \approx^{\mathrm{M} / \xi}[b]_{\xi}=\theta(a, b) } & =\xi(a, b) \wedge \xi(b, a) \\
& =[a]_{\xi} \preccurlyeq^{\mathrm{M} / \xi}[b]_{\xi} \wedge[b]_{\xi} \preccurlyeq^{\mathrm{M} / \xi}[a]_{\xi},
\end{aligned}
$$

i.e., $\approx^{\mathrm{M} / \xi}=\preccurlyeq^{\mathrm{M} / \xi} \cap \succcurlyeq^{\mathrm{M} / \xi}$. In addition to that, (7) follows by (26) and (9) follows by (27). Finally, Theorem 2 yields (8).

Remark 3. Let us note that $\mathbf{M}_{T}$ in Example 3 may be seen as a factorization of $\mathbf{M}=\left\langle L^{Y}, \preccurlyeq^{\mathbf{M}}, \cup^{\mathbf{M}}, 0_{Y}, 1_{Y}\right\rangle$, where $A \preccurlyeq^{\mathbf{M}} B=S(B, A)$ and $\left(A \cup^{\mathbf{M}} B\right)(y)=$ $A(y) \vee B(y)$ (a union of $\mathbf{L}$-sets defined componentwise using $\vee$ in $\mathbf{L}$ ). In particular, we may take a compatible $\mathbf{L}$-preorder $\xi$ induced by $T$, i.e., $\xi(A, B)=$ $\|A \Rightarrow B\|_{T}$. Under this notation, $\mathbf{M}_{T}$ coincides with $\mathbf{M} / \xi$. 
Given an $\mathbf{L}$-preorder $\xi$ which is compatible with $\mathbf{M}$, we may introduce a surjective map $h_{\xi}: M \rightarrow M / \xi$ by putting

$$
h_{\xi}(a)=[a]_{\xi}
$$

The map is called a natural homomorphism induced by $\xi$ and it is indeed a homomorphism which is in addition surjective. Thus, factor algebras with $\mathbf{L}$ orders are homomorphic images. Moreover, for a homomorphism $h: \mathbf{M} \rightarrow \mathbf{N}$, we may introduce a binary L-relation $\xi_{h}$ on $M$ by putting

$$
\xi_{h}(a, b)=h(a) \preccurlyeq^{\mathbf{N}} h(b) .
$$

Analogously as in case of algebras with L-equalities [4, one may check that $\xi_{h}$ is a compatible $\mathbf{L}$-preorder on $\mathbf{M}$ and $\mathbf{M} / \xi_{h}$ can be embedded into $\mathbf{N}$. Namely, one can introduce an embedding $g: \mathbf{M} / \xi_{h} \rightarrow \mathbf{N}$ by putting $g\left([a]_{\xi_{h}}\right)=h(a)$ and show that $h=h_{\xi_{h}} \circ g$. In addition, if $h$ is surjective then $g$ is an isomorphism, cf. [4, Theorem 3.8]. As a result, homomorphic images of $\mathbf{M}$ are isomorphic to factor algebras of $\mathbf{M}$ which is a desirable property.

\section{Varieties of Algebras with Fuzzy Order}

In this section, we provide an analogy of the ordered variety theorem by Bloom [9]. We prove an assertion showing that classes of algebras with fuzzy order defined by graded inequalities are exactly the classes of algebras with fuzzy order closed under the formations of subalgebras, homomorphic images, and direct products.

We start with proving the closure properties of classes definable by graded inequalities. Collections of graded inequalities are formalized as $\mathbf{L}$-sets of formulas. In a more detail, an atomic formula $t \preccurlyeq t^{\prime}$ where $t, t^{\prime} \in T(X)$ are terms, is called an inequality. Then, an $\mathbf{L}$-set $\Sigma$ of inequalities, called a theory, can be seen as a representation of a collection of graded inequalities using terms in $T(X)$. Indeed, for each $t, t^{\prime} \in T(X), \Sigma$ prescribes a degree $\Sigma\left(t \preccurlyeq t^{\prime}\right)$ which can be interpreted as a lower bound of a degree to which $t \preccurlyeq t^{\prime}$ shall be satisfied in a model. Clearly, the standard understanding of theories as sets of formulas can 
be viewed as a particular case of the concept of theories as $\mathbf{L}$-sets of formulas since $\Sigma\left(t \preccurlyeq t^{\prime}\right)=1$ prescribes that $t \preccurlyeq t^{\prime}$ shall be satisfied (fully) in a model of $\Sigma$ and $\Sigma\left(t \preccurlyeq t^{\prime}\right)=0$ means that in a model of $\Sigma$ the inequality $t \preccurlyeq t^{\prime}$ need not be satisfied at all. In other words, $\Sigma\left(t \preccurlyeq t^{\prime}\right)=0$ puts no constraint on models with respect to the satisfaction of $t \preccurlyeq t^{\prime}$.

In order to define models precisely, we utilize the following notation. For an algebra $\mathbf{M}$ with $\mathbf{L}$-order, any map $v: X \rightarrow M$ is called an $\mathbf{M}$-valuation of variables in $X$, i.e., the result $v(x)$ is the value of $x$ in $\mathbf{M}$ under $v$. As in the ordinary case, $v$ admits a unique homomorphic extension $v^{\sharp}$ to $\mathbf{T}(X)$. Thus, $v^{\sharp}(x)=v(x)$ for all $x \in X$ and $v^{\sharp}$ is a map $v^{\sharp}: \mathbf{T}(X) \rightarrow \mathbf{M}$. For $t \in T(X)$, the value $v^{\sharp}(t)$ is called the value of $t$ in $\mathbf{M}$ under $v$. Note that in the literature on fuzzy logics in the narrow sense [1, 3, 21, $v^{\sharp}(t)$ is often denoted by $\|t\|_{\mathbf{M}, v}$. Now, for any inequality $t \preccurlyeq t^{\prime}$, we may introduce the degree to which $t \preccurlyeq t^{\prime}$ is true in $\mathbf{M}$ under $v$ by

$$
\left\|t \preccurlyeq t^{\prime}\right\|_{\mathbf{M}, v}=v^{\sharp}(t) \preccurlyeq^{\mathbf{M}} v^{\sharp}\left(t^{\prime}\right)
$$

and the degree to which $t \preccurlyeq t^{\prime}$ is true in $\mathbf{M}$ (under all $\mathbf{M}$-valuations):

$$
\left\|t \preccurlyeq t^{\prime}\right\|_{\mathbf{M}}=\bigwedge_{v: X \rightarrow M}\left\|t \preccurlyeq t^{\prime}\right\|_{\mathbf{M}, v} .
$$

Under this notation, we call $\mathbf{M}$ a model of $\Sigma$ if $\Sigma\left(t \preccurlyeq t^{\prime}\right) \leq\left\|t \preccurlyeq t^{\prime}\right\|_{\mathbf{M}}$ for all $t, t^{\prime} \in T(X)$ and denote the class of all models of $\Sigma$ by $\operatorname{Mod}(\Sigma)$. That is,

$$
\operatorname{Mod}(\Sigma)=\left\{\mathbf{M} ; \Sigma\left(t \preccurlyeq t^{\prime}\right) \leq\left\|t \preccurlyeq t^{\prime}\right\|_{\mathbf{M}} \text { for all } t, t^{\prime} \in T(X)\right\}
$$

A class $\mathcal{K}$ of algebras with $\mathbf{L}$-orders is called an inequational class if there is $\Sigma$ such that $\mathcal{K}=\operatorname{Mod}(\Sigma)$. Furthermore, if $\mathcal{K}$ is a class of algebras with $\mathbf{L}$-orders, we define a degree to which $t \preccurlyeq t^{\prime}$ is true in (all algebras in) $\mathcal{K}$ by

$$
\left\|t \preccurlyeq t^{\prime}\right\|_{\mathcal{K}}=\bigwedge_{\mathbf{M} \in \mathcal{K}}\left\|t \preccurlyeq t^{\prime}\right\|_{\mathbf{M}}
$$

In particular, for $\mathcal{K}=\operatorname{Mod}(\Sigma),\left\|t \preccurlyeq t^{\prime}\right\|_{\operatorname{Mod}(\Sigma)}$ defined by [38) is called a degree to which $t \preccurlyeq t^{\prime}$ is semantically entailed by $\Sigma$. 
Remark 4. The way we consider theories as $\mathbf{L}$-sets of formulas, define models of theories, and define degrees of semantic entailment is consistent with Pavelka's general approach to abstract logic semantics $[24,25,26$. Clearly, if $\mathbf{M}$ is the trivial algebra with $\mathbf{L}$-order, then $\left\|t \preccurlyeq t^{\prime}\right\|_{\mathbf{M}}=1$ for any $t, t^{\prime} \in T(X)$. In contrast, $\left\|t \preccurlyeq t^{\prime}\right\|_{\mathbf{T}(X)}=1$ if $t=t^{\prime}$ and $\left\|t \preccurlyeq t^{\prime}\right\|_{\mathbf{T}(X)}=0$ otherwise, i.e., all non-trivial inequalities are satisfied in $\mathbf{T}(X)$ to a zero degree.

The following assertion shows the basic closure properties of $\operatorname{Mod}(\Sigma)$. The proof of the assertion uses analogous arguments as in the case of equational classes, i.e., the classes of algebras with fuzzy equalities defined by $\mathbf{L}$-sets of identities [2]. We therefore present only a sketch of the proof, omitting technical details.

Theorem 5. Let $\Sigma$ be an $\mathbf{L}$-set of inequalities. Then, $\operatorname{Mod}(\Sigma)$ is closed under formations of subalgebras, homomorphic images, and direct products.

Proof. The fact that $\operatorname{Mod}(\Sigma)$ is closed under formations of subalgebras is immediate since $\Sigma\left(t \preccurlyeq t^{\prime}\right) \leq\left\|t \preccurlyeq t^{\prime}\right\|_{\mathbf{M}} \leq\left\|t \preccurlyeq t^{\prime}\right\|_{\mathbf{N}}$ for each subalgebra $\mathbf{N}$ of $\mathbf{M} \in \operatorname{Mod}(\Sigma)$. Analogously, if $\mathbf{M}_{i} \in \operatorname{Mod}(\Sigma)(i \in I)$, we may use $\Sigma\left(t \preccurlyeq t^{\prime}\right) \leq$ $\bigwedge_{i \in I}\left\|t \preccurlyeq t^{\prime}\right\|_{\mathbf{M}_{i}} \leq\left\|t \preccurlyeq t^{\prime}\right\|_{\prod_{i \in I} \mathbf{M}_{i}}$ to prove that $\prod_{i \in I} \mathbf{M}_{i} \in \operatorname{Mod}(\Sigma)$. Indeed, in this case we may use the fact that for each $\prod_{i \in I} \mathbf{M}_{i}$-valuation $v$ there are $\mathbf{M}_{i}$-valuations $v_{i}(i \in I)$ such that $v_{i}(x)=(v(x))(i)$ for all $x \in X$ and $i \in I$ and thus $v_{i}^{\sharp}(t)=\left(v^{\sharp}(t)\right)(i)$ for all $t \in T(X)$ and $i \in I$, cf. [3].

Finally, let $\mathbf{M} \in \operatorname{Mod}(\Sigma)$, take a surjective homomorphism $h: \mathbf{M} \rightarrow \mathbf{N}$, and consider an $\mathbf{N}$-valuation $v$. Since $h$ is surjective, there is an $\mathbf{M}$-valuation $w$ such that $v(x)=h(w(x))$ for all $x \in X$, i.e., $v=w \circ h$ with $\circ$ defined as in Remark 2. By induction on the rank of terms, 20 can be used to prove that 
$w^{\sharp} \circ h=(w \circ h)^{\sharp}$. Now, using (21), it follows that

$$
\begin{aligned}
\left\|t \preccurlyeq t^{\prime}\right\|_{\mathbf{M}, w} & =w^{\sharp}(t) \preccurlyeq^{\mathbf{M}} w^{\sharp}\left(t^{\prime}\right) \\
& \leq h\left(w^{\sharp}(t)\right) \preccurlyeq^{\mathbf{N}} h\left(w^{\sharp}\left(t^{\prime}\right)\right) \\
& =\left(w^{\sharp} \circ h\right)(t) \preccurlyeq^{\mathbf{N}}\left(w^{\sharp} \circ h\right)\left(t^{\prime}\right) \\
& =(w \circ h)^{\sharp}(t) \preccurlyeq^{\mathbf{N}}(w \circ h)^{\sharp}\left(t^{\prime}\right) \\
& =v^{\sharp}(t) \preccurlyeq^{\mathbf{N}} v^{\sharp}\left(t^{\prime}\right)=\left\|t \preccurlyeq t^{\prime}\right\|_{\mathbf{N}, v},
\end{aligned}
$$

showing $\Sigma\left(t \preccurlyeq t^{\prime}\right) \leq\left\|t \preccurlyeq t^{\prime}\right\|_{\mathbf{M}} \leq\left\|t \preccurlyeq t^{\prime}\right\|_{\mathbf{N}}$. Hence, $\mathbf{N} \in \operatorname{Mod}(\Sigma)$.

Using the standard terminology in general algebra, a class of algebras with fuzzy orders is called a variety if it is closed under formations of subalgebras, homomorphic images, and direct products. In addition, by $\mathrm{V}(\mathcal{K})$ we denote the variety generated by $\mathcal{K}$, i.e., $\mathrm{V}(\mathcal{K})$ is the least variety containing each algebra with fuzzy order in $\mathcal{K}$. The previous theorem states that each inequational class is a variety. We now turn our attention to the opposite direction of this observation.

Analogously as in the ordinary case, in order to prove that a variety $\mathcal{K}$ is an inequational classes, we utilize particular algebras in $\mathcal{K}$ which may be regarded as the greatest images of $\mathbf{T}(X)$ in $\mathcal{K}$. In particular, for a class $\mathcal{K}$ of algebras with $\mathbf{L}$-order and for a set $X$ of variables, we define

$$
\Phi_{\mathcal{K}}(X)=\left\{\xi_{h} ; h: \mathbf{T}(X) \rightarrow \mathbf{M} \text { for some } \mathbf{M} \in \mathcal{K}\right\},
$$

i.e. $\Phi_{\mathcal{K}}(X)$ is the set of all compatible $\mathbf{L}$-preorders on $\mathbf{T}(X)$ induced by homomorphisms from $\mathbf{T}(X)$ to algebras in $\mathcal{K}$, see (34). Using the same arguments as in [4, Theorem 3.1], the set of all compatible $\mathbf{L}$-preorders on $\mathbf{T}(X)$ is closed under arbitrary intersections. Thus, $\xi_{\mathcal{K}}(X)=\bigcap \Phi_{\mathcal{K}}(X)$ is a compatible $\mathbf{L}$-preorder on $\mathbf{T}(X)$. The corresponding factor algebra

$$
\mathbf{F}_{\mathcal{K}}(\bar{X})=\mathbf{T}(X) / \xi_{\mathcal{K}}(X)
$$

is called the $\mathcal{K}$-free algebra with $\mathbf{L}$-order (generated by $X$ ). The following assertions show properties of $\mathbf{F}_{\mathcal{K}}(\bar{X})$. 
Lemma 6. If $\mathcal{K}$ is variety, then $\mathbf{F}_{\mathcal{K}}(\bar{X}) \in \mathcal{K}$.

Proof. We prove the assertion by showing that $\mathbf{F}_{\mathcal{K}}(\bar{X})$ is isomorphic to a subalgebra of a direct product of algebras $\mathbf{T}(X) / \xi$ in $\mathcal{K}$. Consider a map $h$ of the form $h: T(X) / \xi_{\mathcal{K}}(X) \rightarrow \prod_{\xi \in \Phi_{\mathcal{K}}(X)} T(X) / \xi$ such that

$$
\left(h\left([t]_{\xi_{\mathcal{K}}(X)}\right)\right)(\xi)=[t]_{\xi}
$$

for all $t \in T(X)$ and $\xi \in \Phi_{\mathcal{K}}(X)$. First, the map is well defined since (41) does not depend on the choice of $t$ in $[t]_{\xi_{\mathcal{K}}(X)}$. In order to see that, observe that for $t^{\prime} \in[t]_{\xi_{\mathcal{K}}(X)}$ we have $\left(\xi_{\mathcal{K}}(X)\right)\left(t, t^{\prime}\right)=\left(\xi_{\mathcal{K}}(X)\right)\left(t^{\prime}, t\right)=1$ which means $\xi\left(t, t^{\prime}\right)=\xi\left(t^{\prime}, t\right)=1$ for all $\xi \in \Phi_{\mathcal{K}}(X)$ and thus $[t]_{\xi}=\left[t^{\prime}\right]_{\xi}$. Moreover, $h$ is compatible with functions: Take an $n$-ary $f$, terms $t_{1}, \ldots, t_{n}$ and observe that for all $\xi \in \Phi_{\mathcal{K}}(X)$, we have

$$
\begin{aligned}
& \left(h\left(f^{\mathbf{F}_{\mathcal{K}}(\bar{X})}\left(\left[t_{1}\right]_{\xi_{\mathcal{K}}(X)}, \ldots,\left[t_{n}\right]_{\xi_{\mathcal{K}}(X)}\right)\right)\right)(\xi) \\
& =\left(h\left(\left[f\left(t_{1}, \ldots, t_{n}\right)\right]_{\xi_{\mathcal{K}}(X)}\right)\right)(\xi) \\
& =\left[f\left(t_{1}, \ldots, t_{n}\right)\right]_{\xi} \\
& =f^{\mathbf{T}(X) / \xi}\left(\left[t_{1}\right]_{\xi}, \ldots,\left[t_{n}\right]_{\xi}\right) \\
& =f^{\mathbf{T}(X) / \xi}\left(\left(h\left(\left[t_{1}\right]_{\xi_{\mathcal{K}}(X)}\right)\right)(\xi), \ldots,\left(h\left(\left[t_{n}\right]_{\xi_{\mathcal{K}}(X)}\right)\right)(\xi)\right) \\
& =\left(f^{\prod_{\xi \in \Phi_{\mathcal{K}}(X)} \mathbf{T}(X) / \xi}\left(h\left(\left[t_{1}\right]_{\xi_{\mathcal{K}}(X)}\right), \ldots, h\left(\left[t_{n}\right]_{\xi_{\mathcal{K}}(X)}\right)\right)\right)(\xi) .
\end{aligned}
$$

Therefore, $h$ satisfies 20 . In order to finish the proof, it suffices to check 23). Using (18), for any $t, t^{\prime} \in T(X)$ it follows that

$$
\begin{aligned}
& {[t]_{\xi_{\mathcal{K}}(X)} \preccurlyeq \mathbf{F}^{\mathbf{F}_{\mathcal{K}}(\bar{X})}\left[t^{\prime}\right]_{\xi_{\mathcal{K}}(X)}=\left(\xi_{\mathcal{K}}(X)\right)\left(t, t^{\prime}\right)} \\
& =\bigwedge_{\xi \in \Phi_{\mathcal{K}}(X)} \xi\left(t, t^{\prime}\right) \\
& =\bigwedge_{\xi \in \Phi_{\mathcal{K}}(X)}[t]_{\xi} \preccurlyeq^{\mathbf{T}(X) / \xi}\left[t^{\prime}\right]_{\xi} \\
& =\bigwedge_{\xi \in \Phi_{\mathcal{K}}(X)}\left(h\left([t]_{\xi_{\mathcal{K}}(X)}\right)\right)(\xi) \preccurlyeq \mathbf{T}(X) / \xi\left(h\left(\left[t^{\prime}\right]_{\xi_{\mathcal{K}}(X)}\right)\right)(\xi)
\end{aligned}
$$

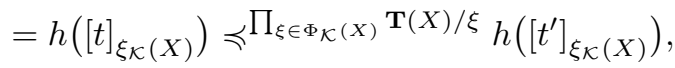

which proves that $h$ is an embedding. Therefore, $\mathbf{F}_{\mathcal{K}}(\bar{X}) \in \mathcal{K}$. 
The following lemma shows that if $\mathcal{K}$ is a variety, then the natural homomorphism $h_{\xi_{\mathcal{K}}(X)}: \mathbf{T}(X) \rightarrow \mathbf{F}_{\mathcal{K}}(\bar{X})$ defined by 33 is a sur-reflection of $\mathbf{T}(X)$ in $\mathcal{K}$. As a consequence, $\mathbf{F}_{\mathcal{K}}(\bar{X})$ can be seen as the greatest image of $\mathbf{T}(X)$ in $\mathcal{K}$, see [5].

Lemma 7. Let $\mathcal{K}$ be a variety, $\mathbf{M} \in \mathcal{K}$, and let $h: \mathbf{T}(X) \rightarrow \mathbf{M}$ be a homomorphism. Then, there is a unique homomorphism $g_{h}: \mathbf{F}_{\mathcal{K}}(\bar{X}) \rightarrow \mathbf{M}$ such that $h=h_{\xi_{\mathcal{K}}(X)} \circ g_{h}$.

Proof. Consider $g_{h}$ given by

$$
g_{h}\left([t]_{\xi_{\mathcal{K}}(X)}\right)=h(t)
$$

for all terms $t \in T(X)$. Note that $g_{h}$ is well defined. Indeed, for $t^{\prime} \in[t]_{\xi_{\mathcal{K}}(X)}$, we have $\xi_{\mathcal{K}}(X)\left(t, t^{\prime}\right)=\xi_{\mathcal{K}}(X)\left(t^{\prime}, t\right)=1$. Since $\xi_{h} \in \Phi_{\mathcal{K}}(X)$, we get $\xi_{h}\left(t, t^{\prime}\right)=$ $\xi_{h}\left(t^{\prime}, t\right)=1$ which yields $h(t) \preccurlyeq^{\mathbf{M}} h\left(t^{\prime}\right)=h\left(t^{\prime}\right) \preccurlyeq^{\mathbf{M}} h(t)=1$ and so $h(t)=h\left(t^{\prime}\right)$, i.e., the values of $g_{h}$ do not depend on the terms selected from the classes $[\cdots]_{\xi_{\mathcal{K}}(X)}$. Obviously, $h=h_{\xi_{\mathcal{K}}(X)} \circ g_{h}$. We show that $g_{h}$ is a homomorphism. For any $n$-ary $f$ and $t_{1}, \ldots, t_{n} \in T(X)$, we have

$$
\begin{aligned}
g_{h}\left(f^{\mathbf{F}_{\mathcal{K}}(\bar{X})}\left(\left[t_{1}\right]_{\xi_{\mathcal{K}}(X)}, \ldots,\left[t_{n}\right]_{\xi_{\mathcal{K}}(X)}\right)\right) & =g_{h}\left(\left[f\left(t_{1}, \ldots, t_{n}\right)\right]_{\xi_{\mathcal{K}}(X)}\right) \\
& =h\left(f\left(t_{1}, \ldots, t_{n}\right)\right) \\
& =f^{\mathbf{M}}\left(h\left(t_{1}\right), \ldots, h\left(t_{n}\right)\right) \\
& =f^{\mathrm{M}}\left(g_{h}\left(\left[t_{1}\right]_{\xi_{\mathcal{K}}(X)}\right), \ldots, g_{h}\left(\left[t_{n}\right]_{\xi_{\mathcal{K}}(X)}\right)\right) .
\end{aligned}
$$

It remains to show that $g_{h}$ is compatible with the $\mathbf{L}$-orders in $\mathbf{F}_{\mathcal{K}}(\bar{X})$ and $\mathbf{M}$. By definition of $g_{h}$, it suffices to prove

$$
\left(\xi_{\mathcal{K}}(X)\right)\left(t, t^{\prime}\right)=[t]_{\xi_{\mathcal{K}}(X)} \preccurlyeq^{\mathbf{F}_{\mathcal{K}}(\bar{X})}\left[t^{\prime}\right]_{\xi_{\mathcal{K}}(X)} \leq h(t) \preccurlyeq^{\mathbf{M}} h\left(t^{\prime}\right)=\xi_{h}\left(t, t^{\prime}\right)
$$

which is indeed the case because $\xi_{h} \in \Phi_{\mathcal{K}}(X)$. Note that since $h_{\xi_{\mathcal{K}}(X)}$ is surjective, $g_{h}$ is determined uniquely.

The following lemma is a consequence of the previous observations and shows that each variety $\mathcal{K}$ of algebras with $\mathbf{L}$-order is generated by a single $\mathcal{K}$-free 
algebra with $\mathbf{L}$-order. Namely, it is sufficient to consider $\mathbf{F}_{\mathcal{K}}(\bar{X})$ generated by a denumerable set $X$ of variables.

Lemma 8. If $\mathcal{K}$ is variety, then $\mathcal{K}=\mathrm{V}\left(\left\{\mathbf{F}_{\mathcal{K}}(\bar{X})\right\}\right)$ where $X$ is denumerable.

Proof. Take $\mathbf{M} \in \mathcal{K}$ and let $[N]_{\mathbf{M}}$ denote the subalgebra of $\mathbf{M}$ which is generated by $N \subseteq M$. We first show that each finitely generated subalgebra of $\mathbf{M}$ is an image of $\mathbf{F}_{\mathcal{K}}(\bar{X})$ where $X$ is denumerable.

Let $N \subseteq M$ be finite and take any surjective map $h: X \rightarrow N$. The map can be extended to a surjective homomorphism $h^{\sharp}: \mathbf{T}(X) \rightarrow[N]_{\mathbf{M}}$. By Lemma 7 there is a homomorphism $g_{h^{\sharp}}: \mathbf{F}_{\mathcal{K}}(\bar{X}) \rightarrow[N]_{\mathbf{M}}$ such that $h^{\sharp}=h_{\xi_{\mathcal{K}}(X)} \circ g_{h^{\sharp}}$. In addition, the surjectivity of $g_{h^{\sharp}}$ follows directly by the surjectivity of $h^{\sharp}$. Hence, $[N]_{\mathbf{M}} \in \mathrm{V}\left(\left\{\mathbf{F}_{\mathcal{K}}(\bar{X})\right\}\right)$.

We continue the proof by showing that $\mathbf{M}$ can be embedded into a factorization of a subalgebra of a direct product of its finitely generated subalgebras. In the proof, we use the following notation: We put

$$
\mathcal{M}=\{N \neq \emptyset ; N \subseteq M \text { and } N \text { is finite }\}
$$

and consider the direct product $\prod_{N \in \mathcal{M}}[N]_{\mathbf{M}}$. For any $a \in \prod_{N \in \mathcal{M}}[N]_{\mathbf{M}}$, we write $a^{\supseteq N}=m$ if the following condition is satisfied: $N \in \mathcal{M}$ and for each $N^{\prime} \in \mathcal{M}$ such that $N \subseteq N^{\prime}$, we have $a\left(N^{\prime}\right)=m$. Now, let

$$
P=\left\{a \in \prod_{N \in \mathcal{M}}[N]_{\mathbf{M}} ; a^{\supseteq N}=m \text { for some } N \in \mathcal{M} \text { and } m \in M\right\} .
$$

Clearly, $P$ is a non-empty subuniverse of the direct product of all finitely generated subalgebras of $\mathbf{M}$. Furthermore, we may introduce a binary L-relation $\xi$ on $\mathbf{P}$ as follows:

$$
\xi(a, b)=m \preccurlyeq^{\mathrm{M}} n
$$

where $m, n \in M$ such that $a \supseteq N=m$ and $b \supseteq N=n$ for some $N \in \mathcal{M}$. Observe that such $N$ always exists and $m, n \in M$ are given uniquely. Thus, $\xi$ is well defined. In addition to that, $\xi$ is a compatible $\mathbf{L}$-preorder on $\mathbf{P}$ : Reflexivity of $\xi$ is obvious; in order to see that $\xi$ is $\otimes$-transitive, consider $a, b, c \in P$ and 
let $\xi(a, b)=m \preccurlyeq^{\mathrm{M}} n$ for $a^{\supseteq N}=m$ and $b^{\supseteq N}=n$ and $\xi(b, c)=p \preccurlyeq^{\mathrm{M}} q$ for $b^{\supseteq N^{\prime}}=p$ and $c^{\supseteq N^{\prime}}=q$. Thus, for $N \cup N^{\prime}$, we have $a \supseteq N \cup N^{\prime}=m$, $c^{\supseteq N \cup N^{\prime}}=q$, and $b^{\supseteq N \cup N^{\prime}}=n=p$. Hence, $\xi(a, b) \otimes \xi(b, c)=m \preccurlyeq^{\mathrm{M}}$ $n \otimes n \preccurlyeq^{\mathrm{M}} q \leq m \preccurlyeq^{\mathrm{M}} q=\xi(a, c)$. Analogously one may check that $\xi$ is compatible with all functions in $\mathbf{P}$. Moreover, $\preccurlyeq^{\mathbf{P}} \subseteq \xi$ follows directly by the fact that $\mathbf{P}$ is a subalgebra of $\prod_{N \in \mathcal{M}}[N]_{\mathbf{M}}$.

Finally, consider $h: M \rightarrow P / \xi$ such that $h(m)=[a]_{\xi}$ for $a \supseteq N=m$. Clearly, $h$ is well defined since for $b \supseteq N^{\prime}=m$, we have $\xi(a, b)=\xi(b, a)=1$ and thus $[a]_{\xi}=[b]_{\xi}$. Now, take an $n$-ary function $f^{\mathbf{M}}$ and $m_{1}, \ldots, m_{n} \in M$. We have

$$
f^{\mathbf{P} / \xi}\left(h\left(m_{1}\right), \ldots, h\left(m_{n}\right)\right)=f^{\mathbf{P} / \xi}\left(\left[a_{1}\right]_{\xi}, \ldots,\left[a_{n}\right]_{\xi}\right)=\left[f^{\mathbf{P}}\left(a_{1}, \ldots, a_{n}\right)\right]_{\xi},
$$

where $a_{1}^{\supseteq N_{1}}=m_{1}, \ldots, a_{n}^{\supseteq N_{n}}=m_{n}$. Observe that for $a=f^{\mathbf{P}}\left(a_{1}, \ldots, a_{n}\right)$ we have $a \supseteq N_{1} \cup \ldots \cup N_{n}=f^{\mathbf{M}}\left(m_{1}, \ldots, m_{n}\right)$ and thus

$$
f^{\mathbf{P} / \xi}\left(h\left(m_{1}\right), \ldots, h\left(m_{n}\right)\right)=\left[f^{\mathbf{P}}\left(a_{1}, \ldots, a_{n}\right)\right]_{\xi}=h\left(f^{\mathbf{M}}\left(m_{1}, \ldots, m_{n}\right)\right) .
$$

We now show that $h$ is an embedding. Take $m, n \in M$ and consider $a, b \in P$ such that $a \supseteq N=m$ and $b \supseteq N^{\prime}=n$, respectively. Since for $N \cup N^{\prime}$ we have $a \supseteq N \cup N^{\prime}=m$ and $b \supseteq N \cup N^{\prime}=n$, it follows that

$$
m \preccurlyeq^{\mathrm{M}} n=\xi(a, b)=[a]_{\xi} \preccurlyeq^{\mathrm{P} / \xi}[b]_{\xi}=h(m) \preccurlyeq^{\mathrm{P}} h(n) .
$$

Hence, the map $h: \mathbf{M} \rightarrow \mathbf{P} / \xi$ defined as above is an embedding. As a consequence, we have $\mathbf{M} \in \mathrm{V}\left(\left\{[N]_{\mathbf{M}} ; N \in \mathcal{M}\right\}\right) \subseteq \mathrm{V}\left(\left\{\mathbf{F}_{\mathcal{K}}(\bar{X})\right\}\right)$.

An important property of $\mathcal{K}$-free algebras with $\mathbf{L}$-order is that they satisfy exactly all inequalities which are satisfied by all algebras in the variety $\mathcal{K}$. This pertains not only to inequalities which are satisfied fully (i.e., to degree 1) but to all inequalities which may be satisfied to arbitrary degrees in $\mathbf{L}$. Indeed, as a consequence of the following lemma, we obtain that $\left\|t \preccurlyeq t^{\prime}\right\|_{\mathcal{K}}=\left\|t \preccurlyeq t^{\prime}\right\|_{\mathbf{F}_{\mathcal{K}}(\bar{X})}$ for all $t, t^{\prime} \in T(X)$.

Lemma 9. Let $\mathcal{K}$ be a variety. For any $t, t^{\prime} \in T(X)$, we have

$$
\left\|t \preccurlyeq t^{\prime}\right\|_{\mathcal{K}}=\left(\xi_{\mathcal{K}}(X)\right)\left(t, t^{\prime}\right) .
$$


Proof. Observe that the $\leq$-part of $(42)$ follows directly by the fact that $\mathbf{F}_{\mathcal{K}}(\bar{X}) \in$ $\mathcal{K}$. In order to prove the $\geq$-part, take $\mathbf{M} \in \mathcal{K}$ and an $\mathbf{M}$-valuation $v: X \rightarrow M$. The valuation admits a homomorphic extension $v^{\sharp}: \mathbf{T}(X) \rightarrow \mathbf{M}$ for which $\left\|t \preccurlyeq t^{\prime}\right\|_{\mathbf{M}, v}=v^{\sharp}(t) \preccurlyeq^{\mathbf{M}} v^{\sharp}\left(t^{\prime}\right)=\xi_{v^{\sharp}}\left(t, t^{\prime}\right)$. Since $\xi_{v^{\sharp}} \in \Phi_{\mathcal{K}}(X)$, we get $\xi_{\mathcal{K}}(X) \subseteq$ $\xi_{v^{\sharp}}$ and thus $\left\|t \preccurlyeq t^{\prime}\right\|_{\mathbf{M}, v} \geq\left(\xi_{\mathcal{K}}(X)\right)\left(t, t^{\prime}\right)$, proving the $\geq$-part of 42 .

The following assertion shows that each variety of algebras with fuzzy orders is an inequational class of a theory over a denumerable set of variables. The assertion is a direct analogy of the Bloom variety theorem [9] of ordered algebras.

Theorem 10. If $\mathcal{K}$ is a variety, then there is $\Sigma$ such that $\mathcal{K}=\operatorname{Mod}(\Sigma)$.

Proof. Let $X$ be a denumerable set of variables and put $\Sigma=\operatorname{Ineq}_{X}(\mathcal{K})$ where $\left(\operatorname{Ineq}_{X}(\mathcal{K})\right)\left(t \preccurlyeq t^{\prime}\right)=\left\|t \preccurlyeq t^{\prime}\right\|_{\mathcal{K}}$ for all $t, t^{\prime} \in T(X)$. By standard arguments, $\mathcal{K} \subseteq \operatorname{Mod}\left(\operatorname{Ineq}_{X}(\mathcal{K})\right)=\operatorname{Mod}(\Sigma)$ and

$$
\operatorname{Ineq}_{X}(\mathcal{K})=\operatorname{Ineq}_{X}\left(\operatorname{Mod}\left(\operatorname{Ineq}_{X}(\mathcal{K})\right)\right)=\operatorname{Ineq}_{X}(\operatorname{Mod}(\Sigma))
$$

i.e., $\left\|t \preccurlyeq t^{\prime}\right\|_{\mathcal{K}}=\left\|t \preccurlyeq t^{\prime}\right\|_{\operatorname{Mod}(\Sigma)}$ for all $t, t^{\prime} \in T(X)$. Therefore, using 42, we get $\xi_{\mathcal{K}}(X)=\xi_{\operatorname{Mod}(\Sigma)}(X)$, i.e., $\mathbf{F}_{\mathcal{K}}(\bar{X})$ coincides with $\mathbf{F}_{\operatorname{Mod}(\Sigma)}(\bar{X})$. Hence, Lemma 8 yields $\mathcal{K}=\operatorname{Mod}(\Sigma)$.

As a consequence of the previous observations, we get that varieties are exactly inequational classes:

Corollary 11. A class $\mathcal{K}$ of algebras with $\mathbf{L}$-orders is a variety iff $\mathcal{K}$ is an inequational class.

In the rest of this paper, we comment of further closure properties of inequational classes defined by theories using only particular degrees in $\mathbf{L}$. The comments are inspired by an approach to algebras in fuzzy setting proposed in 22] and which later appeared in [12]. The major difference between [22] and the Pavelka-style approaches (including the approach in the present paper) is that [22] allows to consider algebras with fuzzy equalities using all BL-algebras taken as structures of truth degrees at the same time. As a consequence, the 
approach uses equational theories in the usual sense (ordinary sets of identities) and exploits the fact that varieties of algebras with fuzzy equalities can be put in correspondence with varieties of ordinary algebras by means of skeletons and homomorphic images.

Analogous characterization as in 22 can also be established in our case with a fixed $\mathbf{L}$ as the structure of degrees. Indeed, for each algebra $\mathbf{M}=$ $\left\langle M, \preccurlyeq^{\mathbf{M}}, F^{\mathbf{M}}\right\rangle$ with $\mathbf{L}$-order, let ske(M) denote the algebra with $\mathbf{L}$-order defined on $M$ with the same functions as in $\mathbf{M}$, and with $\preccurlyeq^{\text {ske(M) }}$ being the identity L-relation. Note that each $\mathbf{M}$ is a homomorphic image of ske(M) which is called the skeleton of $\mathbf{M}$. A theory $\Sigma$ is crisp if $\Sigma\left(t \preccurlyeq t^{\prime}\right) \in\{0,1\}$ for all $t, t^{\prime} \in T(X)$. We may now establish the following characterization.

Theorem 12. A class $\mathcal{K}$ of algebras with $\mathbf{L}$-orders is a variety closed under formations of sekeletons iff $\mathcal{K}=\operatorname{Mod}(\Sigma)$ for crisp $\Sigma$.

Proof. Obviously, if $\mathcal{K}=\operatorname{Mod}(\Sigma)$ for crisp $\Sigma$, then $\mathcal{K}$ is closed under formations of sekeletons. Conversely, let $\mathcal{K}$ be a variety closed under skeletons. Observe that if $\left\|t \preccurlyeq t^{\prime}\right\|_{\mathcal{K}}<1$, then there is $\mathbf{M} \in \mathcal{K}$ and $v: X \rightarrow M$ such that $\left\|t \preccurlyeq t^{\prime}\right\|_{\mathbf{M}, v}<$ 1. Now, for the sekeleton $\operatorname{ske}(\mathbf{M})$ of $\mathbf{M}$ and using the same valuation $v$, we have $\left\|t \preccurlyeq t^{\prime}\right\|_{\operatorname{ske}(\mathbf{M}), v}=0$. Therefore, $\left\|t \preccurlyeq t^{\prime}\right\|_{\mathcal{K}}=0$ because ske( $\left.\mathbf{M}\right) \in \mathcal{K}$. As a consequence, $\operatorname{Ineq}_{X}(\mathcal{K})$ is crisp and we have $\mathcal{K}=\operatorname{Mod}(\Sigma)$ for $\operatorname{crisp} \Sigma=$ $\operatorname{Ineq}_{X}(\mathcal{K})$, see Theorem 10

Remark 5. Theorem 12 may further be generalized. For instance, for each $c<1$, one may consider for $\mathbf{M} \in \mathcal{K}$ an algebra $\mathbf{M}^{c}$ with the same universe and functions as $\mathbf{M}$ and with $\preccurlyeq^{\mathbf{M}^{c}}$ defined by

$$
a \preccurlyeq \mathbf{M}^{c} \quad b= \begin{cases}1, & \text { if } a \preccurlyeq^{\mathbf{M}} b=1, \\ c, & \text { otherwise, }\end{cases}
$$

for all $a, b \in M$. Obviously, $\mathbf{M}^{c}$ is an algebra with $\mathbf{L}$-order. Using analogous arguments as in the proof of Theorem 12 , we conclude that the following statements are equivalent: 
(i) $\mathcal{K}$ is a variety such that $\mathbf{M}^{c} \in \mathcal{K}$ whenever $\mathbf{M} \in \mathcal{K}$;

(ii) $\mathcal{K}=\operatorname{Mod}(\Sigma)$ where $\left\{\Sigma\left(t \approx t^{\prime}\right) ; t, t^{\prime} \in T(X)\right\} \subseteq[0, c] \cup\{1\}$.

Clearly, for $c=0$ we obtain exactly the equivalence in Theorem 12 Further investigation of closure properties of varieties of algebras with $\mathbf{L}$-order and the corresponding constraints on $\Sigma$ should prove interesting.

\section{Acknowledgment}

Supported by grant no. P202/14-11585S of the Czech Science Foundation.

\section{References}

[1] Radim Belohlavek, Fuzzy Relational Systems: Foundations and Principles, Kluwer Academic Publishers, Norwell, MA, USA, 2002.

[2] _ Birkhoff variety theorem and fuzzy logic, Archive for Mathematical Logic 42 (2003), no. 8, 781-790.

[3] Radim Belohlavek and Vilem Vychodil, Fuzzy Equational Logic, Studies in Fuzziness and Soft Computing, vol. 186, Springer, 2005.

[4] _ Algebras with fuzzy equalities, Fuzzy Sets and Systems 157 (2006), no. 2, 161201.

[5] _ Fuzzy Horn logic II, Archive for Mathematical Logic 45 (2006), no. 2, 149-177.

[6] - Formal concept analysis and linguistic hedges, International Journal of General Systems 41 (2012), no. 5, 503-532.

[7] _ Attribute dependencies for data with grades, CoRR abs/1402.2071 (2014), http://arxiv.org/abs/1402.2071

[8] Garrett Birkhoff, On the structure of abstract algebras, Proc. Cambridge Phil. Soc. 31 (1935), 433-454.

[9] Stephen L. Bloom, Varieties of ordered algebras, Journal of Computer and System Sciences 13 (1976), no. 2, 200-212.

[10] Ulrich Bodenhofer, Bernard De Baets, and János Fodor, A compendium of fuzzy weak orders: Representations and constructions, Fuzzy Sets and Systems 158 (2007), no. 8, 811-829.

[11] Stanley Burris and H. P. Sankappanavar, A Course in Universal Algebra, SpringerVerlag, New York, 1981. 
[12] Petr Cintula and Petr Hájek, Triangular norm based predicate fuzzy logics, Fuzzy Sets and Systems 161 (2010), no. 3, 311-346.

[13] Petr Cintula, Petr Hájek, and Carles Noguera (eds.), Handbook of Matematical Fuzzy Logic, Volume 1, Studies in Logic, Mathematical Logic and Foundations, vol. 37, College Publications, 2011.

[14] Petr Cintula, Petr Hájek, and Carles Noguera (eds.), Handbook of Matematical Fuzzy Logic, Volume 2, Studies in Logic, Mathematical Logic and Foundations, vol. 38, College Publications, 2011.

[15] Francesc Esteva and Lluís Godo, Monoidal t-norm based logic: Towards a logic for leftcontinuous t-norms, Fuzzy Sets and Systems 124 (2001), no. 3, 271-288.

[16] Francesc Esteva, Lluís Godo, and Carles Noguera, A logical approach to fuzzy truth hedges, Information Sciences 232 (2013), 366-385.

[17] Nikolaos Galatos, Peter Jipsen, Tomacz Kowalski, and Hiroakira Ono, Residuated Lattices: An Algebraic Glimpse at Substructural Logics, Volume 151, 1st ed., Elsevier Science, San Diego, USA, 2007.

[18] Giangiacomo Gerla, Fuzzy Logic. Mathematical Tools for Approximate Reasoning, Kluwer Academic Publishers, Dordrecht, The Netherlands, 2001.

[19] Joseph A. Goguen, The logic of inexact concepts, Synthese 19 (1979), 325-373.

[20] Siegfried Gottwald, Mathematical fuzzy logics, Bulletin of Symbolic Logic 14 (2008), no. $2,210-239$.

[21] Petr Hájek, Metamathematics of Fuzzy Logic, Kluwer Academic Publishers, Dordrecht, The Netherlands, 1998.

[22] _ A note on Birkhoff variety theorem in fuzzy logic, preprint, ICS AV ČR, 2002.

[23] Erich Peter Klement, Radko Mesiar, and Endre Pap, Triangular Norms, 1 ed., Springer, 2000.

[24] Jan Pavelka, On fuzzy logic I: Many-valued rules of inference, Mathematical Logic Quarterly 25 (1979), no. 3-6, 45-52.

[25] _ On fuzzy logic II: Enriched residuated lattices and semantics of propositional calculi, Mathematical Logic Quarterly 25 (1979), no. 7-12, 119-134.

[26] _ On fuzzy logic III: Semantical completeness of some many-valued propositional calculi, Mathematical Logic Quarterly 25 (1979), no. 25-29, 447-464.

[27] Vilem Vychodil, Direct limits and reduced products of algebras with fuzzy equalities, Multiple-Valued Logic and Soft Computing 13 (2007), no. 1-2, 1-28.

[28] Lotfi A. Zadeh, A fuzzy-set-theoretic interpretation of linguistic hedges, Journal of Cybernetics 2 (1972), no. 3, 4-34. 